\title{
COCYCLES' STABILITY FOR PARTIALLY HYPERBOLIC SYSTEMS
}

\author{
A. Katok And A. Kononenko
}

\begin{abstract}
In this paper we establish Livshitz-type theorems for partially hyperbolic systems. To be more precise, we prove that for a large class of partially hyperbolic transformations and flows the subspace of Hoelder coboundaries is closed and can be described by some natural geometric conditions. This class includes an open, in $C^{2}$ topology, neighborhood of the time-one maps of contact Anosov flows (for example, the geodesic flows on manifolds of negative curvature).

Along the way we prove several results on the transitivity of the pair of stable and unstable foliations for partially hyperbolic systems. In particular, we establish the transitivity property for the time-one maps of contact Anosov flows and their small perturbations, which has important applications to the stable ergodicity of the time-one maps of geodesic flows on the manifolds of negative curvature.
\end{abstract}

\section{Stability of cocycle spaces}

Let $f$ be a transformation of a space $X$. The cohomological equation corresponding to a fixed function $\phi$ (also called a cocycle) is the following equation, with an unknown function $h$,

$$
\phi(x)-h(x)+h(f(x))=0, x \in X .
$$

If this equation has solutions then $\phi$ is called a coboundary, and $h$ is called a transfer function. Two functions are called cohomologous if their difference is a coboundary. Depending on the classes of regularity of cocycles and transfer functions allowed one associates with the transformation $f$ various cohomology spaces ([10], [13]).

A number of well known results describes the spaces of coboundaries for different types of maps $f$. The most celebrated results of this kind are the Livshitz-type theorems for hyperbolic diffeomorphisms and flows ([4], [7], [8], [10] (Section 19.2), [13], [14], [15], [16]). However some non-hyperbolic systems also display certain regularity for cocycles. These include Diophantine translations of a torus, affine maps ([12], Section 10.5), partially

Received December 27, 1995.

The first author is partially supported by NSF Grant DMS 9404061. 
hyperbolic toral automorphisms ([20]), integrable systems, area-preserving flows on surfaces of high genus ([5]).

It is a simple and very general fact that under some natural conditions the closure of the space of coboundaries is equal to the intersection of the kernels of all $f$-invariant linear functionals. It is true, for example, for $C^{\infty}$-cocycles with $C^{\infty}$ transfer functions, for Hoelder cocycles with Hoelder transfer functions, etc (the proof is similar to the proof of Proposition 9.12 in [12]). Therefore, if we want to describe a certain space of coboundaries $B$ for a map $f$ we naturally encounter the following two questions:

(1) Is $B$ closed, and thus equal to the intersection of the kernels of all $f$-invariant functionals?

(2) What is the structure of the set $\mathcal{F}$ of all $f$-invariant functionals? In particular, is it possible to find a dense or generating subset of $\mathcal{F}$ that consists of the functionals which have "nice descriptions" in terms of the dynamics of $f$ ?

Livshitz theorems describe the space of coboundaries as being the common zero set for a family of functionals corresponding to the periodic points of $f$, where $f$ is a hyperbolic diffeomorphism, thus giving a positive answer to both questions.

In the present paper, we give an affirmative answer to the first question for a large class of partially hyperbolic dynamical systems. Moreover, we show that for such systems the subspace of Hoelder coboundaries can be described as a common zero set of some natural geometrically defined functionals - the periodic cycles functionals (see Definition 3.3), thus answering the second question.

Before we proceed to the formulations of our results we would like to introduce some convenient terminology (which is a slight modification of the terminology first suggested in [12]).

Let $\mathcal{P}$ be a space of functions on a space $X$ endowed with some topology. Let $\mathcal{P}_{1}$ be another space of functions (not necessarily equipped with any topology).

Definition 1.1. The space of $\mathcal{P}$-cocycles, i.e., cocycles which lie in $\mathcal{P}$, of a transformation $f$ of $X$ is called $\mathcal{P}_{1}$-stable if the space of the cocycles cohomologous to a constant, with the transfer functions from $\mathcal{P}_{1}$, is closed with respect to the topology of $\mathcal{P}$.

With the exception of Section 6, we will work with Hoelder cocycles. To be more precise, the role of $\mathcal{P}$ will be played by the space $L_{\alpha}-$ the space of Hoelder functions with the fixed exponent $\alpha \in(0,1]$. The role of $\mathcal{P}_{1}$ will be played by $L_{\beta}, \beta \in(0,1]$ or the space of continuous functions. 
We will refer to the functions from $L_{\alpha}$ as $\alpha$-Hoelder functions, and we will refer to the functions from $L_{1}$ as Lipschitz functions.

\section{Transitivity of a pair of foliations}

Let $M$ be a Riemannian manifold and $S$ its submanifold. For $x, y \in M$ (correspondingly $x, y \in S$ ) we will denote by $d_{M}(x, y)$ (correspondingly $\left.d_{S}(x, y)\right)$ the infinum of the lengths of the smooth curves in $M$ (correspondingly $S$ ) connecting $x$ and $y$.

Definition 2.1. A pair of continuous foliations $\mathcal{F}_{1}$ and $\mathcal{F}_{2}$ with smooth leaves is called locally transitive if, for any compact subset $M_{1}$ of $M$, there exists $N \in \mathbb{N}$, such that for any $\epsilon>0$ there exists $\delta>0$ such that for every $x, y \in M$ with $x \in M_{1}$ and $d_{M}(x, y)<\delta$ there are points $x_{1}, \ldots, x_{N} \in M$, satisfying the following conditions:

(1) $x_{1}=x, x_{N}=y$;

(2) $x_{i+1} \in \mathcal{F}_{j}\left(x_{i}\right), i=1, \ldots, N-1, j=1$ or 2 ;

(3) $d_{M}\left(x_{i}, x\right) \leq \epsilon$ and $d_{\mathcal{F}_{j}\left(x_{i}\right)}\left(x_{i+1}, x_{i}\right)<2 \epsilon, i=1, \ldots, N-1, j=1$ or 2 .

The notion of local transitivity of a pair of foliations was introduced by Brin and Pesin in [2]. They studied extensively the local transitivity property for the pair of stable and unstable foliations for partially hyperbolic dynamical systems, its relations to other properties of the system, and its stability under perturbations. An important class of partially hyperbolic dynamical systems - the extensions of the hyperbolic ones - was proved to posses the transitivity property (slightly weaker property then the local transitivity) generically, by Brin in [3]. Brin's examples include, in particular, the frame flows on the manifolds of negative curvature. Also, the transitivity condition appears in the work of Grayson, Pugh and Shub [6] on stable ergodicity. Namely, it is one of the conditions which they require for the system to be stably ergodic.

The following definition is the Hoelder version of local transitivity:

Definition 2.2. A pair of continuous foliations $\mathcal{F}_{1}$ and $\mathcal{F}_{2}$ with smooth leaves is called locally $\alpha$-Hoelder transitive if there exists $N \in \mathbb{N}, \delta>0$, $C>0$ such that for every $x$ and $y \in M$ with $d_{M}(x, y)<\delta$ there are points $x_{1}, \ldots, x_{N} \in M$, satisfying the following conditions:

(1) $x_{1}=x, x_{N}=y$;

(2) $x_{i+1} \in \mathcal{F}_{j}\left(x_{i}\right) i=1, \ldots, N-1, j=1$ or 2 ;

(3) $d_{\mathcal{F}_{j}\left(x_{i}\right)}\left(x_{i+1}, x_{i}\right)<C d_{M}(x, y)^{\alpha}, i=1, \ldots, N-1, j=1$ or 2 .

The following definition is useful in questions related to Lipschitz cocycles, in particular, to $C^{\infty}$-cocycles. 
Definition 2.3. A pair of continuous foliations $\mathcal{F}_{1}$ and $\mathcal{F}_{2}$ with smooth leaves is called weakly locally $\alpha$-Hoelder transitive, where $\alpha \in(0,1]$, if there exists $\delta>0$ and $C>0$ such that for every $x, y \in M$ with $d_{M}(x, y)<$ $\delta$ there are points $x_{1}, \ldots, x_{k} \in M$, satisfying the following conditions:

(1) $x_{1}=x, x_{k}=y$;

(2) $x_{i+1} \in \mathcal{F}_{j}\left(x_{i}\right), i=1, \ldots, k-1, j=1$ or 2 ;

(3) $\sum_{j=1}^{j=k-1} d_{\mathcal{F}_{j}\left(x_{i}\right)}\left(x_{i+1}, x_{i}\right)<C d_{M}(x, y)^{\alpha}$.

Clearly, local $\alpha$-Hoelder transitivity implies weak local $\alpha$-Hoelder transitivity and transitivity.

\section{Stability theorems for Hoelder cocycles}

Recall the following standard definition due to Brin and Pesin ([2]):

Definition 3.1. A diffeomorphism $g$ of a manifold $M$ with a Riemannian norm $\|\cdot\|$ is called partially hyperbolic if there exist real numbers $\lambda_{1}>$ $\mu_{1}>0, i=1,2, K, K^{\prime}>0$ and a continuous splitting of the tangent bundle

$$
T M=E^{+} \bigoplus E^{0} \bigoplus E^{-}
$$

such that for all $x \in M$, for all $v \in E^{+}(x)\left(v \in E^{+}(x)\right.$ respectively) and $n>0$ ( $n<0$ respectively) we have for the differential $g_{*}: T M \rightarrow T M$

$$
\left\|g_{*}(v)\right\| \leq K e^{-\lambda_{1} n}|| v \|\left(\left\|g_{*}(v)\right\| \leq K e^{-\lambda_{2}|n|}\|v\| \text {, respectively }\right)
$$

and for all $n \in \mathbb{Z}$ and $v \in E^{0}(x)$ we have

$$
\left\|g_{*}(v)\right\| \geq K^{\prime} e^{-\mu_{1} n}\|v\|, n>0 \text { and }\left\|g_{*}(v)\right\| \geq K^{\prime} e^{-\mu_{2}|n|}\|v\|, n<0 .
$$

Furthermore, we assume that the distribution $E^{0}$ is uniquely integrable. We will call $E^{+}$and $E^{-}$stable and unstable distributions respectively.

Note that if $M$ is compact these notions do not depend on the ambient Riemannian metric.

The following fact is a direct corollary of the Hadamard-Perron theorem (see, for example, [10], Theorem 6.2.8).

Theorem A 3.0.1. For a partially hyperbolic dynamical system, there are Hoelder foliations $W^{s}$ and $W^{u}$ tangent to the distributions $E^{+}$and $E^{-}$ respectively. We call this foliations stable and unstable foliations. The individual leaves of these foliations are $C^{\infty}$-immersed submanifolds of $M$, and are called stable and unstable manifolds.

For the basic theory of the partially hyperbolic systems see [2] and [9]. 
Definition 3.2. We will call a set $C$ of points $x_{1}, x_{2}, \ldots, x_{2 n}, x_{2 n+1}=$ $x_{1} \in M a$ periodic cycle if $x_{2 k} \in W^{s}\left(x_{2 k-1}\right)$ and $x_{2 k+1} \in W^{u}\left(x_{2 k}\right)$, for $k=1, \ldots, n$.

Notice that if $y \in W^{s}(x)$ then $d_{M}\left(f^{i}(x), f^{i}(y)\right)$ decreases exponentially, thus $\phi\left(f^{i}(x)\right)-\phi\left(f^{i}(y)\right)$ also decreases exponentially. Therefore, the series

$$
P^{+}(x, y)(\phi)=\sum_{i=0}^{\infty}\left(\phi\left(f^{i}(x)\right)-\phi\left(f^{i}(y)\right)\right)
$$

converges absolutely for any Hoelder function $\phi$. Similarly, if $y \in W^{u}(x)$ then the series

$$
P^{-}(x, y)(\phi)=-\sum_{i=-1}^{-\infty}\left(\phi\left(f^{i}(x)\right)-\phi\left(f^{i}(y)\right)\right)
$$

converges absolutely for any Hoelder function $\phi$.

Let us notice that if $\phi$ is $C^{\infty}$ then $P^{+}(x, y)$ and $P^{-}(x, y)$ are infinitely differentiable with respect to $y$ along the stable and unstable foliations, and the derivatives are continuous with respect to $x$.

Definition 3.3. For a periodic cycle $C$, we will denote by $F(C)$ the following continuous functional on the space $L$ of Hoelder functions:

$$
\begin{gathered}
F(C)(\phi)=P^{+}\left(x_{1}, x_{2}\right)(\phi)+P^{-}\left(x_{2}, x_{3}\right)(\phi)+\cdots \\
+P^{+}\left(x_{2 n-1}, x_{2 n}\right)(\phi)+P^{-}\left(x_{2 n}, x_{1}\right)(\phi) .
\end{gathered}
$$

We will call this functional a periodic cycle functional.

The following results give general criteria for cocycle's stability for partially hyperbolic diffeomorphisms:

Theorem 3.1. If $f$ is a partially hyperbolic diffeomorphism such that the pair $\left(W^{s}, W^{u}\right)$ is locally transitive, then, for any $\beta \in(0,1]$, the space of $\beta$-Hoelder cocycles of $f$ is $C^{0}$-stable, and the subspace of cocycles cohomologous to a constant is the common zero set of the periodic cycles functionals, i.e., $\phi \in L_{\beta}$ is cohomologous to a constant, with $C^{0}$ transfer function, if and only if $F(C)(\phi)=0$ for all periodic cycles $C$.

Remark. Notice that if the cocycle is $C^{\infty}$ and the periodic cycles functionals vanish then the transfer function constructed in Theorem 3.1 has continuous derivatives of all orders along the stable and unstable foliations. 
Theorem 3.2. If $f$ is a partially hyperbolic diffeomorphism such that the pair $\left(W^{s}, W^{u}\right)$ is locally $\alpha$-Hoelder transitive, then, for any $\beta \in(0,1]$, the space of $\beta$-Hoelder cocycles is both $\alpha \beta$-Hoelder stable and $C^{0}$-stable.

If the pair $\left(W^{s}, W^{u}\right)$ is weakly locally $\alpha$-Hoelder transitive then the space of Lipschitz cocycles is $C^{0}$-stable and $\alpha$-Hoelder stable.

In all cases, the subspace of cocycles cohomologous to a constant is the common zero set of the periodic cycles functionals.

Proof of Theorems 3.1 and 3.2. Let $\phi$ be a $\beta$-Hoelder function. Then there exists a constant $L$ such that $|\phi(a)-\phi(b)| \leq L d_{M}(a, b)^{\beta}$, for all $a, b \in M$.

Assume that $F(C)(\phi)=0$ for any periodic cycle $C$.

We will call a set $S(x, y)$ of points $x_{1}=x, x_{2}, \ldots, x_{k}=y \in M$ a broken path from $x$ to $y$, if $x_{i+1} \in \mathcal{F}_{j}\left(x_{i}\right), i=1, \ldots, k-1, j=1$ or 2 . We will call $\sum_{j=1}^{j=k-1} d_{\mathcal{F}_{j}\left(x_{i}\right)}\left(x_{i+1}, x_{i}\right)$ the length of the broken path $S$. We will call the points $x_{i}$ the turning points of $S$ and the points $x$ and $y$ the end points of $S$. We will also say that $S$ connects $x$ with $y$.

Let

$$
F_{1}(S(x, y))(\phi)=\sum_{i=1}^{k-1} P^{*}\left(x_{i}, x_{i+1}\right)(\phi),
$$

where $*$ is equal to + or - depending on whether the $x_{i+1}$ belong to, correspondingly, the stable or unstable manifolds of $x_{i}$.

Fix an arbitrary point $x \in M, \quad$ and define a function $h(y)=F_{1}(S(x, y))(\phi)$, where $S$ is some broken path from $x$ to $y$. The function $h(y)$ is defined for all points $y \in M$ due to the transitivity of the pair $\left(W^{s}, W^{u}\right)$, and $h(y)$ does not depend on the choice of the path $S$, due to the fact that $F(C)(\phi)=0$, for any periodic cycle $C$.

Now we will prove that due to the local transitivity condition (correspondingly local $\alpha$-Hoelder transitivity condition) on the pair of stable and unstable foliations, $h(y)$ is continuous, (correspondingly $\alpha \beta$-Hoelder).

Indeed, there exists $\delta_{1}>0$ such that for $a$ and $b$ belonging to the same leaf $W$ of the stable foliation with $d_{W}(a, b)<\delta_{1}$,

$$
\begin{aligned}
P^{+}(a, b)(\phi)= & \sum_{i=0}^{\infty}\left(\phi\left(f^{i}(a)\right)-\phi\left(f^{i}(b)\right)\right) \leq \sum_{i=0}^{\infty} L d_{M}\left(f^{i}(a), f^{i}(b)\right)^{\beta} \leq \\
& \sum_{i=0}^{\infty} L \lambda^{i \beta} d_{M}(a, b)^{\beta}=K_{1} d_{M}(a, b)^{\beta},
\end{aligned}
$$

where $\lambda$ is a contracting exponent of the stable foliation, and $L$ and $K_{1}=$ $\sum_{i=0}^{\infty} L \lambda^{i \beta}$ are constants that depend on $\phi$ and $f$ but not $a$ and $b$. 
Similarly, there exists $\delta_{2}>0$ such that for $a$ and $b$ belonging to the same leaf $W$ of the unstable foliation with $d_{W}(a, b)<\delta_{2}$,

$$
P^{-}(a, b)(\phi) \leq K_{2} d_{M}(a, b)^{\beta},
$$

for some constant $K_{2}$ that depends on $\phi$ and $f$ but not $a$ and $b$.

Let $K=\max \left(K_{1}, K_{2}\right)$. Then we have

$$
P^{*}(a, b)(\phi) \leq K d_{M}(a, b)^{\beta},
$$

for all $a$ and $b$ which belong to the same leaf of either the stable or unstable foliation and such that $d_{M}(a, b)<\min \left(\delta_{1}, \delta_{2}\right)$.

Suppose the pair $\left(W^{s}, W^{u}\right)$ is locally transitive. Fix $\epsilon>0$ and let $\delta$ and $N \in \mathbb{N}$ be as in Definition 2.1. Also, assume that $\delta<\min \left(\delta_{1}, \delta_{2}\right)$. Let $z_{1}$ and $z_{2}$ be arbitrary points in $M$ such that $d_{M}\left(z_{1}, z_{2}\right)<\delta$, and let $Z=\left\{x_{1}=z_{1}, x_{2}, \ldots, x_{N}=z_{2}\right\}$ be a broken path from $z_{1}$ to $z_{2}$ as in Definition 2.1. Then,

$$
h\left(z_{1}\right)-h\left(z_{2}\right)=F_{1}(Z)(\phi) \leq \sum_{i=1}^{N-1} K d_{M}\left(x_{i}, x_{i+1}\right)^{\beta} \leq(N-1) K(2 \epsilon)^{\beta},
$$

which proves that $h$ is continuous.

If the pair $\left(W^{s}, W^{u}\right)$ is locally $\alpha$-Hoelder transitive then we have

$$
\begin{aligned}
h\left(z_{1}\right)-h\left(z_{2}\right)=F_{1}(Z)(\phi) & \leq \sum_{i=1}^{N-1} K d_{M}\left(x_{i}, x_{i+1}\right)^{\beta} \\
& \leq(N-1) K C^{\beta} d_{M}\left(z_{1}, z_{2}\right)^{\alpha \beta},
\end{aligned}
$$

where $C$ is as in Definition 2.2, which shows that $h$ is $\alpha \beta$-Hoelder.

If $\phi$ is Lipschitz, and the pair $W^{s}, W^{u}$ is weakly locally $\alpha$-Hoelder transitive then we still have

$$
h\left(z_{1}\right)-h\left(z_{2}\right)=F_{1}(Z)(\phi) \leq \sum_{i=1}^{k-1} K d_{M}\left(x_{i}, x_{i+1}\right) \leq K C d_{M}\left(z_{1}, z_{2}\right)^{\alpha},
$$

which shows that $h$ is $\alpha$-Hoelder.

Now we will show that $-h(y)$ solves the cohomological equation for $\phi$. Indeed, let $S(x, y)=\left\{x_{1}=x, x_{2}, \ldots, x_{k}=y\right\}$ be some broken path from point $x$ to point $y$, and let $S(f(x), f(y))$ be a broken path from $f(x)$ to 
$f(y)$ consisting of points $f\left(x_{1}\right)=f(x), f\left(x_{2}\right), \ldots, f\left(x_{k}\right)=f(y)$. Then

$$
\begin{aligned}
h(f(y))= & h(f(x))+F_{1}(S(f(x), f(y))(\phi)= \\
& h(f(x))+\sum_{i=1}^{k-1} P^{*}\left(f\left(x_{i}\right), f\left(x_{i+1}\right)\right)(\phi)= \\
& h(f(x))+\sum_{i=1}^{k-1} P^{*}\left(x_{i}, x_{i+1}\right)(\phi)-\sum_{i=1}^{k-1}\left(\phi\left(x_{i}\right)-\phi\left(x_{i+1}\right)\right)= \\
& h(f(x))+\sum_{i=1}^{k-1} P^{*}\left(x_{i}, x_{i+1}\right)(\phi)-\phi(x)+\phi(y)= \\
& h(f(x))+h(y)-\phi(x)+\phi(y) .
\end{aligned}
$$

If we denote $h(f(x))+\phi(x)$ by $c$, then we have

$$
\phi(y)+h(y)-h(f(y))=c .
$$

This shows that if $F(C)(\phi)=0$ for any periodic cycle $C$ then $\phi$ is cohomologous to a constant cocycle.

Now assume that $\phi(y)-h(y)+h(f(y))=c$ is cohomologous to a constant with the continuous transfer function $h$. Let $C=\left\{x_{1}, \ldots, x_{2 k}, x_{2 k+1}=x_{1}\right\}$ be some periodic cycle.

Notice that

$$
\begin{aligned}
& P^{+}\left(x_{i}, x_{i+1}\right)(\phi)=\sum_{j=0}^{\infty}\left(\phi\left(f^{j}\left(x_{i}\right)\right)-\phi\left(f^{j}\left(x_{i+1}\right)\right)\right)= \\
& \sum_{j=0}^{\infty}\left(h\left(f^{j}\left(x_{i}\right)\right)-h\left(f^{j+1}\left(x_{i}\right)\right)+c-\left(h\left(f^{j}\left(x_{i+1}\right)\right)-h\left(f^{j+1}\left(x_{i+1}\right)\right)+c\right)\right)= \\
& \sum_{j=0}^{\infty}\left(\left(h\left(f^{j}\left(x_{i}\right)\right)-h\left(f^{j}\left(x_{i+1}\right)\right)\right)-\left(h\left(f^{j+1}\left(x_{i}\right)\right)-h\left(f^{j+1}\left(x_{i+1}\right)\right)\right)=\right. \\
& h\left(x_{i}\right)-h\left(x_{i+1}\right)-\lim _{j \rightarrow \infty}\left(h\left(f^{j}\left(x_{i}\right)\right)-h\left(f^{j}\left(x_{i+1}\right)\right)\right)=h\left(x_{i}\right)-h\left(x_{i+1}\right),
\end{aligned}
$$

if $i$ is odd.

Similarly,

$$
P^{-}\left(x_{i}, x_{i+1}\right)(\phi)=h\left(x_{i}\right)-h\left(x_{i+1}\right),
$$

if $i$ is even.

Therefore,

$$
F(C)(\phi)=\sum_{i=1}^{i=2 k}\left(h\left(x_{i}\right)-h\left(x_{i+1}\right)\right)=0 .
$$

Theorems 3.1 and 3.2 are proved. 
Notice, that from the last part of the proof we get the following

Corollary 3.1. For any partially hyperbolic diffeomorphism, if a Hoelder function $\phi$ is cohomologous to a constant with a continuous transfer function then it belongs to the intersection of the kernels of all periodic cycles functionals.

\section{Transformations coming from the Weyl chamber flows}

Recall the following standard

Definition 4.1. A pair of smooth foliations $\mathcal{F}_{1}$ or $\mathcal{F}_{2}$ is called totally nonintegrable with index $p \in \mathbb{N}$ if for any $x \in M$ the Lie brackets of degree at most $p$ of the vector fields tangent to either $\mathcal{F}_{1}$ or $\mathcal{F}_{2}$ span the whole $T_{x} M$.

We will call a smooth distribution $E$ on $M$ totally non-integrable with index $p \in \mathbb{N}$ if for any $x \in M$ the Lie brackets of degree at most $p$ of the vector fields tangent to $E$ span the whole $T_{x} M$.

The following simple statement opens up a way to construct a large class of examples of transformations with Hoelder stable and $C^{0}$-stable spaces of Hoelder cocycles:

Proposition 4.1. If a pair of $C^{\infty}$ foliations on $M$ is totally non-integrable with index $p$, then it is locally transitive and, if $M$ is compact it is locally $\left(1 / 2^{p}\right)$-Hoelder transitive.

We omit the proof of Proposition 4.1, since it is standard and is unrelated to our main subject here. (A proof of a similar result that can be easily modified to prove Proposition 4.1 can be found in [2].)

From Proposition 4.1 and Theorem 3.2 we have the following

Corollary 4.1. Let $G$ be a semisimple group of non-compact type, $A$ its maximal split Cartan subgroup, $\Gamma$ an irreducible cocompact lattice, and $K$ any compact subgroup of $G$ that commutes with $A$. Then, for any $\beta \in$ $(0,1]$, for any regular element $a \in A$ acting on $M=K \backslash G / \Gamma$, the space of $\beta$-Hoelder cocycles is both $\beta / 2^{p}$-Hoelder stable and $C^{0}$-stable, where $p$ depends only on $G$ and $K$, but not on $a, \Gamma$ or $\beta$.

In particular, if $K$ is maximal such compact group as described above then the space of $\beta$-Hoelder cocycles is $\beta / 2$-Hoelder stable.

If $a \in A$ is not regular but $\log (a)$ still has non-trivial projections to all simple components of the Lie algebra $\mathcal{G}$ of $G$, then the space of $\beta$ Hoelder cocycles is both $\beta / 2^{p(a)}$-Hoelder stable and $C^{0}$-stable, where now $p(a)$ depends only on $G, K$ and $a$, but not on $\Gamma$ or $\beta$.

Moreover, the subspace of cocycles cohomologous to a constant is the common zero set of the periodic cycles functionals. 
In particular, if $K$ is the maximal compact subgroup commuting with $A$, the above action of $a \in A$ is a part of the Weyl chamber flow. And if $G$ is rank one the Weyl chamber flow coincides with the geodesic flow on the corresponding compact rank-one locally symmetric space of non-compact type.

Also notice, that if $\Gamma$ is not cocompact then the $C^{0}$ part of Corollary 4.1 still holds.

Theorem 3.1 together with the results of Brin and Pesin ([2], Theorem 4.3) imply the following

Corollary 4.2. For any transformation $f$ described in Corollary 4.1 there is an open neighborhood $U(f)$ in Dif $f^{2}(M)$ such that for every $f_{1} \in U(f)$ the space of $\beta$-Hoelder cocycles of $f_{1}$ is $C^{0}$-stable, and the subspace of cocycles cohomologous to a constant is the common zero set of the periodic cycles functionals.

\section{Time-one maps of contact Anosov flows}

We will call a continuous distribution $E$ on a manifold $Q$ locally transitive ( $\alpha$-Hoelder locally transitive respectively) if for any $\epsilon>0$ there exists $\delta>0$ such that for any $x, y \in Q$ with $d_{Q}(x, y)<\delta$ there exists a broken piecewise smooth curve $\gamma$ tangent to $E$, on its intervals of smoothness, and such that its length is less then $\epsilon\left(C \delta^{\alpha}\right.$, for some constant $C$, respectively).

Then we prove the following:

Proposition 5.1. If for an Anosov flow $g^{t}$ on a manifold $Q$ the distribution $E^{+} \bigoplus E^{-}$is locally transitive ( $\alpha$-Hoelder locally transitive, respectively) then the pair $\left(W^{s}, W^{u}\right)$ is locally transitive (weakly $\alpha$-Hoelder locally transitive, respectively).

Proof. For $y \in Q$ we will denote the ball of radius $a$ around $y$ on $Q$ by $B_{a}(y)$.

For a point $y \in Q$ denote the orbit passing through $y$ by $O_{y}$.

Fix $\epsilon$. We will find numbers $\delta$ and $N$ satisfying Definition 2.1 in several steps.

Let $x$ be an arbitrary point of $Q$. In steps $1-7 x$ is the same point.

Step 1. There exists $0<\delta_{1}<\epsilon$ such that for any $0<\delta<\delta_{1}$ and any $y \in B_{\delta}(x)$ there is a broken piecewise smooth curve $\gamma$ tangent to $E^{+} \bigoplus E^{-}$ and connecting $x$ and $y$. Moreover, $\gamma$ is inside $B_{\epsilon_{1} / 2}(x)$, (where $\epsilon_{1} / 2=\epsilon / 2$ or $\epsilon_{1} / 2=C_{1} \delta^{\alpha}$, for some constant $C_{1}$. Therefore, approximating $\gamma$ by broken paths we see that for any $\epsilon_{2}$ there exists a broken path inside $B_{\epsilon_{1}}(x)$ connecting $x$ with some point $y_{1} \in B_{\epsilon_{2}}(y)$. (Such an approximation always exists due to the following simple argument. Project the curve $\gamma$ to the transverse section $T$ constructed in Step 2. Let $\Pi(\gamma)$ be the projection on 
$T$. Then due to the product structure on $T$ (see Step 3), for small enough $\delta_{1}$, the curve $\Pi(\gamma)$ can be arbitrarily well approximated by a broken path on $T$, (see Step 2) and the lifts (see Step 3) of such approximations to $Q$, with initial point $x$, give approximations of $\gamma$ by broken paths on $Q$.)

Step 2. Let $0<\delta_{2}$ be so small that there exists a piece $T$ of smooth sub-manifold of $Q$ of codimension one, such that, for $y \in B_{\delta_{2}}(x)$, the maximal connected piece of $O_{y}$ that contains $y$ and belongs entirely to $B_{\delta_{2}}(x)$, intersects $T$ transversely in exactly one point $\Pi(x) \in T$. From now on, for $y \in B_{\delta_{2}}(x)$, we will denote by $O_{y}$ not the whole orbit $O_{y}$ but the maximal connected piece of the orbit that contains $y$ and belongs entirely to $B_{\delta_{2}}(x)$.

We have a well defined projection $\Pi: B_{\delta_{2}}(x) \rightarrow T$ along the orbits. Two points $z_{1}$ and $z_{2}$ are mapped by $\Pi$ into one point $n \in T$ if and only if $O_{z_{1}}=O_{z_{2}}$. Moreover, their stable and unstable manifolds are mapped into the same sub-manifolds of $T$. We will denote these sub-manifolds by $F^{s}(n)$ and $F^{u}(n)$. Therefore, we obtain two local foliations $F^{s}$ and $F^{u}$ in the $\delta_{2}$-neighborhood of $x$ in $T$. We will call broken paths on $T$ defined with respect to $F^{s}$ and $F^{u}$ broken paths on $T$.

Step 3. Locally, there is a product structure on $T$. Namely, there exists $0<\delta_{3}<\delta_{2}$ such that for $n_{1}, n_{2} \in T_{1}=\left(B_{\delta_{3}}(x) \bigcap T\right)$ there are unique points $I_{1}$ and $I_{2}$ of intersections of $F^{s}\left(n_{1}\right)$ and $F^{u}\left(n_{2}\right)$ and of $F^{u}\left(n_{1}\right)$ and $F^{s}\left(n_{2}\right)$ correspondingly.

Moreover, for any $\delta<\delta_{3}$ there exists $\delta^{\prime}$ such that if $n_{1}, n_{2} \in B_{\delta^{\prime}}(x)$ then $I_{1}, I_{2} \in B_{\delta}(x)$.

Now, notice that for any broken path $S_{T_{1}}=\left\{X, n_{1}, \ldots, n_{k}\right\} \in T_{1}$ on $T$ and for any $\tilde{X} \in O_{X}$ there is a unique lift to $Q$, which is a broken path $S_{Q}(\tilde{X})=\left\{\tilde{X}, x_{1}, \ldots, x_{k}\right\} \in Q$ such that $\Pi\left(x_{i}\right)=n_{i}, i=1, \ldots, k$. Indeed, there is a unique point $x_{1}$ of intersection of $W^{*}(\tilde{X})$ with $O_{n_{1}}$. Then there is a unique point $x_{2}$ of intersection of $W^{*}\left(x_{1}\right)$ with $O_{n_{2}}$, and so on, where $*$ is equal to $s$ or $u$ in accordance with the sequence of $s$ and $u$ in the broken path $S_{T_{1}}$ on $T$.

Step 4. Since, $T$ is transversal to the orbits of $g^{t}$, there exist $0<\delta_{4}<\delta_{3}$ such that for any $y \in B_{\delta_{4}}(x)$ the norm of the differential $D\left(\left.\Pi\right|_{W^{*}(y)}\right)$ is bounded away from zero by some constant independent of $y$ and $*=s$ or $u$.

Step 5. Therefore, due to the choice of $\delta_{4}$ in Step 4, there exists $0<\delta_{5}<\delta_{4}$ and a constant $K$ such that for any $\delta<\delta_{5}$ a lift to $Q$ of any broken path on $T$ inside a $\delta$-neighborhood of $x$ belongs to a $K \delta$ neighborhood of $\tilde{x}$ in $Q$, if $\tilde{x} \in O_{x} \cap B_{\delta_{5}}(x)$.

Step 6. Therefore, due to the local product structure (Step 3), the lifting procedure (Step 3) and the restriction on the norm of the differential (Step 4), there exist $0<\delta_{6}<\delta_{5}$ such that for any $y \in B_{\delta_{6}}(x)$ there is a 
broken path consisting of 3 turning points inside $B_{\delta_{5}}(x)$ that connects $y$ with some point $z \in O_{x}$ such that $d_{Q}(y, z)<K d_{Q}(\Pi(y), x)$.

Step 7. Now we will prove that there exists $0<\delta_{7}<\delta_{6}, 0<\delta_{7}<\delta_{1}$, such that for any point of $O_{x} \bigcap B_{\delta_{7}}(x)$ there is a broken path inside $B_{\delta_{5}}$ with no more then some fixed number $L$ of turning points that connects this point with $x$.

First of all notice that if there is a path $S$ inside $B_{\delta_{6}}(x)$ connecting $x$ and $p \in O_{x}$ then for any $p^{\prime} \in O_{x}$ between $x$ and $p$ on $O_{x}$ there is a path $S^{\prime}$ connecting $x$ and $p^{\prime}$, and such that $S^{\prime}$ has as many turning points as $S$ does. Indeed, consider, the closed broken path $\Pi(S)$ in $T$. Due to the local product structure it is easy to construct a continuous family $S^{t}$ of closed broken paths in $T$, with the same number of turning points as $S$ has, and such that $S^{0}=\Pi(S)$ and $S^{1}=\{x\}$, where $\{x\}$ is a trivial broken path that consists of several copies of the point $x$, and the end points for all $S^{t}$ are all equal to the point $x$. Then the end point $p^{t}$ of their lifts $S_{Q}^{t}(x)$ changes continuously on $O_{x}$ and $p^{0}=p$ and $p^{1}=x$.

Let us prove that there are broken paths $S_{1}\left(x, p_{1}\right)$ and $S_{2}\left(x, p_{2}\right)$ such that $p_{1}$ precedes $x$ on $O_{x}$ and $p_{2}$ follows it. Let us construct $S_{1}$, the construction of $S_{2}$ is absolutely parallel.

Fix point any point $y \in O_{x} \bigcap B_{\delta_{6}}(x)$ such that it precedes $x$ and $d_{Q}(y, x)<\left(\frac{\delta_{5}}{2 C}\right)^{2}$. As in Step 1 , let $\gamma$ be a curve tangent to $E^{+} \bigoplus E^{-}$ connecting $x$ and $y$. Let $\epsilon_{2}<\frac{d_{Q}(x, y)}{10 K}$. Then, due to the Step 1, there exists a broken path $S$ in $Q$ inside $B_{\delta_{5}}$ connecting $x$ with some point $y_{1} \in B_{\epsilon_{2}}(y)$. Then, we can add two more points to $S$ in order to connect $y_{1}$ with some point $p_{1} \in O_{x}$. Denote the resulting path by $S_{1}$, then the point $p_{1}$ and the path $S_{1}$ satisfy our requirements. Indeed, $S_{1}$ is inside $B_{\delta_{5}}$ and $d_{Q}\left(p_{1}, x\right)>d_{Q}(y, x)-K d_{Q}\left(y_{1}, y\right)>0$, thus $p_{1}$ precedes $x$ on $O_{x}$.

Now, we will show that it is possible to choose the broken path $S_{1}$ with no more than 6 turning points. Let $L>6$ be the minimal possible number of turning points and let

$$
S_{1}=\left\{x_{1}=x, x_{2}, x_{3}, x_{4}, x_{5}, x_{6}, \ldots, x_{L}=p_{1}\right\}
$$

be the path with exactly $L$ turning points. Let $\left\{x_{4}, y_{5}, y_{6} \in O_{x}\right\}$ be a path like in Step 3, connecting $x_{4}$ with some point $y_{6} \in O_{x}$. Then,

1) If $y_{6}$ precedes $x$ on $O_{x}$, then the broken path $\left\{x_{1}, x_{2}, x_{3}, x_{4}, y_{5}, y_{6}\right\}$ contradicts to the choice of $S_{1}$.

2) If $y_{6}=x$ then the broken path

$$
\left\{y_{6}=x, y_{5}, x_{4}, x_{5}, x_{6}, \ldots, x_{L}=p_{1}\right\}
$$

contradicts to the choice of $S_{1}$. 
3) Assume that $y_{6}$ follows $x$ on $O_{x}$. Then let

$$
\tilde{S}_{1}=\left\{p_{1}=x_{L}, \ldots, x_{6}, x_{5}, x_{4}, y_{5}, y_{6}\right\}
$$

be the path connecting $p_{1}$ and $y_{6}$. Then we consider $\Pi\left(\tilde{S}_{1}\right)$ and construct the family $S^{t}$ of broken paths on $T$, just like before, i.e., $S^{0}=\Pi\left(\tilde{S}_{1}\right)$ and $S^{1}=\{x\}$. Then consider the lifts $S_{Q}^{t}\left(p_{1}\right)$. Denote the end point of $S_{Q}^{t}\left(p_{1}\right)$ by $p^{t}$. Then, $p^{0}=y_{6}$, and $p^{1}=p_{1}$. Therefore, there exists $t_{0}$ such that $p^{t_{0}}=x$. Then, the broken path $S_{Q}^{t_{0}}\left(p_{1}\right)$ contradicts to the choice of $S_{1}$.

Similarly, construct $S_{2}$. And let

$$
\delta_{7}(x)=\delta_{7}=\min \left\{d_{Q}\left(x, p_{1}\right), d_{Q}\left(x, p_{2}\right)\right\} .
$$

Then we see that for any $y \in B_{\delta_{7}}(x)$ there is a broken path inside $B_{\delta_{5}}(x)$ with no more then 8 turning points that connects $x$ and $y$. Therefore, for any $y_{1}, y_{2} \in B_{\delta_{7}}(x)$ there is a broken path inside $B_{\delta_{5}}(x)$ with no more then 16 turning points that connects $y_{1}$ and $y_{2}$.

Step 8. Cover $Q$ by the balls $U_{x}=B_{\delta_{7}(x)}(x)$. Let $U_{1}, \ldots U_{m}$ be some finite sub-cover. Then there exists $\delta_{8}$ such that for any $y \in Q$ there is $i \in\{1, \ldots, m\}$ such that $B_{\delta_{8}}(y) \subset U_{i}$.

Therefore, the conditions of Definition 2.1 are satisfied with $\delta=\delta(\epsilon)=$ $\delta_{8}$ and $N=16$, where $x_{i}$ are the centers of $U_{i}$. (Actually, for the case of contact Anosov flows $N=7$ is enough (easily follows from the arguments in the proof of Theorem 18.3.6 from [10]), but we do not need the estimates on $N$ in this work.)

The weak local $\alpha$-Hoelder transitivity follows easily from the local transitivity of $\left(W^{s}, W^{u}\right)$ and the local $\alpha$-Hoelder transitivity of $E^{+} \bigoplus E^{-}$. Indeed, there exists $\delta>0$ such that for $x, y$ with $d_{Q}(x, y)<\delta$ there is a curve $\gamma$ connecting $x$ and $y$ as in Step 1. Then its length $l$ is less then $C_{2} d_{Q}(x, y)^{\alpha}$, where $C_{2}$ is some constant that does not depend on $x$ and $y$. Let $\epsilon<\min \{l / 10, \delta\}$. Then due to the local transitivity there exists $\delta^{\prime}$ such that every point of $B_{\delta^{\prime}}(y)$ can be connected with $y$ by a broken path of length less then $\epsilon$.

Then, like in Steps 1 and 7, we can approximate $\gamma$ by a broken path $S^{\prime}$ in such a way that the length of $S^{\prime}$ is less then $l+\epsilon$, and $S^{\prime}$ connects $x$ with some point $y^{\prime} \in B_{\delta^{\prime}}(y)$. Then connecting $y^{\prime}$ with $y$ we can extend $S^{\prime}$ to a broken path $S$ that connects $x$ and $y$ and has length less then $l+2 \epsilon<2 C_{2} d_{Q}(x, y)^{\alpha}$.

Then we have

Theorem 5.1. For a contact Anosov flow $g^{t}$ on a compact manifold the pair $\left(W^{s}, W^{u}\right)$ is locally transitive and weakly locally 1/2-Hoelder transitive. 
(The local 1/2-Hoelder transitivity of $E^{+} \bigoplus E^{-}$follows from the fact that $E^{+} \bigoplus E^{-}$is smooth (Lemma 18.3.7, [10]), its total non-integrability (Theorem 18.3.6, [10]) with index 2 and an obvious analog of Proposition 4.1 for a single totally non-integrable distribution.)

Let $M$ be a compact Riemannian manifold of negative curvature, and $g^{t}$ be the geodesic flow on $S M$. It is well known that $g^{t}$ is a contact Anosov flow, and thus Theorem 5.1 applies to $g^{t}$.

As an immediate corollary of Theorems 3.1, 3.2 and 5.1 we obtain the following

Corollary 5.1. For the time-one map of a contact Anosov flow on a compact manifold the space of $\beta$-Hoelder cocycles is $C^{0}$-stable, and the subspace of cocycles cohomologous to a constant is the common zero set of the periodic cycles functionals.

Moreover, the spaces of Lipschitz cocycles and $C^{\infty}$-cocycles are 1/2Hoelder stable, and the subspaces of cocycles cohomologous to a constant are the common zero sets of the periodic cycles functionals.

Now, we will show that small perturbations of the time-one maps of contact Anosov flows on compact manifolds have transitive but not necessarily locally transitive pairs of stable and unstable foliations. Therefore, Theorem 3.1 is not immediately applicable. Nevertheless, we will show that for small enough perturbations the conclusion of Theorem 3.1 still holds, i.e., the space of Hoelder cocycles cohomologous to a constant is the common zero set of the periodic cycles functionals.

Step 1. Cover the manifold $Q$ by a finite number of open sets $U_{i}$ such that inside each $U_{i}$ the conditions described in Steps 2,3 and 4 of the proof of Theorem 5.1 are satisfied. Namely, there are projections $\Pi_{i}: U_{i} \rightarrow T_{i}$, where $T_{i}$ are codimension one submanifolds transversal to the neutral foliation of $g=g^{1}$. Moreover, the images of the foliations $W^{s}$ and $W^{u}$ are foliations $F_{i}^{s}$ and $F_{i}^{u}$ of $T_{i}$, which define a product structure on $T_{i}$ and such that their derivatives satisfy the conditions of Step 4.

Step 2. Let $g^{\prime}$ be a small perturbation of $g$. Then, by Hirsh-PughShub "Fundamental Theorem of Normally Hyperbolic Invariant Manifolds," if $g^{\prime}$ is close enough to $g$ then it is also partially hyperbolic, with one-dimentional neutral foliation $O^{\prime}$, and foliations $W^{s^{\prime}}, W^{u^{\prime}}$, which are $C^{0}$ close to the neutral foliation of $g$ and $W^{s}, W^{u}$, respectively. Therefore, if the perturbation was small enough, then we will have projections $\Pi^{\prime}: U_{i} \rightarrow T_{i}$ along the neutral foliation for $g^{\prime}$. Moreover, the images of the foliations $W^{s^{\prime}}$ and $W^{u^{\prime}}$ are foliations $F_{i}^{s^{\prime}}$ and $F_{i}^{u^{\prime}}$ of $T_{i}$, which define a product structure on $T_{i}$. 
Also, any broken path on $T$ with respect to $F_{i}^{s^{\prime}}$ and $F_{i}^{u^{\prime}}$ may be lifted to a broken path on $Q$ with respect to $W^{s^{\prime}}$ and $W^{u^{\prime}}$, and the lifting is unique up to the choice of the initial point.

Step 3. Now, find $\epsilon$ so small that for any $x \in Q$ there is an $i=i(x)$ such that $B_{2 \epsilon}(x) \subset U_{i}$.

Since $g$ is locally transitive we can find $N$ and $\delta(\epsilon)$ such that every two points $x$ and $y$ such that $d_{Q}(x, y) \leq \delta(\epsilon)$ can be joined by a broken path on $Q$ with respect to $W^{s}$ and $W^{u}$, which is inside $B_{\epsilon}(x)$ and has no more than $N$ turning points.

Step 4. For any $\epsilon_{1}$, there is a neighborhood $V$ of $g$ in $C^{2}$ topology, such that for any $g^{\prime} \in V$, the pair $\left(F_{i}^{s^{\prime}}, F_{i}^{u^{\prime}}\right)$ is so close to $\left(F_{i}^{s}, F_{i}^{u}\right)$ and $\left(W^{s^{\prime}}, W^{u^{\prime}}\right)$ is so close to $\left(W^{s}, W^{u}\right)$ that, for any $i$, and for any broken path $P$ on $T_{i}$, with no more than $N$ turning points, with respect to $F_{i}^{s}$ and $F_{i}^{u}$ there is a broken path $P^{\prime}$ on $T_{i}$ with respect to $F_{i}^{s^{\prime}}$ and $F_{i}^{u^{\prime}}$, with no more than $N$ turning points and such that the distance between the end points of the lifts of $P$ and $P^{\prime}$ is not bigger than $\epsilon_{1}$, if the lifts are with the same initial point.

Step 5. Let us prove that if $g^{\prime}$ is close enough to $g$ then every two points $x$ and $y$ such that $d_{Q}(x, y)<\delta(\epsilon) / 2$ can be joined by a broken path on $Q$ with respect to $W^{s^{\prime}}$ and $W^{u^{\prime}}$, which is inside $B_{2 \epsilon}(x)$ and has no more than $N+4$ turning points. Due to the arguments similar to the ones in the proof of Proposition 5.1 it is enough to show that for $x$ and $y$ from one leaf of $O^{\prime}$ (with no more than $N+2$ turning points, in this case).

Indeed, let $x$ be an arbitrary point of $Q$. And let $y \in O^{\prime}(x)$ and $d_{Q}(x, y)<\delta(\epsilon) / 2$. Let $z \in O^{\prime}(x)$ be such that $d_{Q}(x, z)=\delta(\epsilon)$ and $y$ lies between $x$ and $z$ on $O^{\prime}(x)$. There is a broken path $S$ on $Q$ with respect to $W^{s}$ and $W^{u}$, which is inside $B_{\epsilon}(x)$, has no more than $N$ turning points, and joins $x$ and $z$.

Now, let $i=i(x)$. Consider $P=\Pi_{i}(S)$. Let $P^{\prime}$ be as in Step 4 above. Consider the lift $S^{\prime}$ of $P^{\prime}$ with respect to $W^{s^{\prime}}$ and $W^{u^{\prime}}$ and initial point $x$. Then the distance between the end point $p$ of the lift of $P^{\prime}$ and $z$ is less than $\epsilon_{1}$. Choosing $\epsilon_{1}$ small enough we can guarantee, just like in Step 7 of the proof of Proposition 5.1, that if we add two more turning points to $S^{\prime}$ we will get a broken path on $Q$ with respect to $W^{s^{\prime}}$ and $W^{u^{\prime}}$ connecting $x$ with some point $y_{1}$ on $O^{\prime}(x)$, such that $d_{Q}\left(y_{1}, z\right)<\delta(\epsilon) / 2$. Therefore contracting the path $P^{\prime}$ as in Step 7 of the proof of Proposition 5.1 we can connect $x$ and $y$ by a broken path with respect to $W^{s^{\prime}}$ and $W^{u^{\prime}}$, with no more than $N+2$ turning points.

Also, it is easy to se that if $S$ was inside $B_{\epsilon}(x)$, than for $g$ and $g^{\prime}$ close enough, $S^{\prime}$ is inside $B_{2 \epsilon}(x)$.

Step 6. Choose a neighborhood $U$ of $g$ in $D i f f^{2}(Q)$ such that it would 
satisfy all "close enough" conditions above. We can always do that since $\epsilon, \delta(\epsilon), U_{i}, T_{i}$ and $\Pi_{i}$ are fixed throughout all the steps in our argument.

Thus, we have the following

Proposition 5.2. Every small enough perturbation $g^{\prime}$ of a time-one map $g$ of a contact Anosov flow on a compact manifold $Q$ has a transitive pair of stable and unstable foliations $W^{s^{\prime}}$ and $W^{u^{\prime}}$, i.e., there exists $\epsilon>0$ and $N^{\prime} \in \mathbb{N}$ such that every $x, y \in Q$ can be joined by a broken path with no more then $N^{\prime}$ turning points $\left\{x_{i}\right\}$ and such that $d_{W^{*}\left(x_{i}\right)}\left(x_{i}, x_{i+1}\right)<\epsilon$, $i=1, \ldots N-1$.

Notice that it is impossible to claim local transitivity in Proposition 5.2, since $g$ can always be perturbed so that in a neighborhood of some point $x$ the pair $\left(W^{s^{\prime}}, W^{u^{\prime}}\right)$ will become locally completely integrable (actually that is a generic situation). Therefore, for small enough $\epsilon$, the local transitivity condition will fail.

Transitivity of the pair $\left(W^{s^{\prime}}, W^{u^{\prime}}\right)$ allows us to define a solution $h$ for the cohomological equation as in the proof of Theorem 3.1. (The local transitivity was used only to prove the continuity of the solution.) This solution is uniformly continuous along $W^{s^{\prime}}$ and $W^{u^{\prime}}$. Moreover, it is uniformly continuous with respect to the leaves. To be more precise, for any $\epsilon>0$ there exists $\delta>0$ such that for any $x, y \in Q$ if $y \in W^{*}(x)$ and $d_{W *(x)}(x, y)<\delta$ then $|h(x)-h(y)|<\epsilon$.

Let us prove that it is also continuous along $O^{\prime}$. Fix a point $x$. As we showed above, there is a broken path $S$ that connects $x$ with a point $y$ belonging to $O^{\prime}(x)$ and such that $S$ can be contracted. Therefore, we have a continuous family of paths $S^{t}$ connecting $x$ with all the points of $O^{\prime}(x)$ from some neighborhood of $x$. But then $F_{1}\left(S^{t}\right)(\phi)$ also changes continuously, for any Hoelder function $\phi$. Thus the solution $h$ is continuous along $O^{\prime}$. This, together with the uniform continuity of $h$ on the leaves of $W^{s}$ and $W^{u}$, proves that $h$ is continuous on $Q$.

Thus we have the following:

Theorem 5.2. Let $Q$ be a compact manifold that admits a contact Anosov flow. Then there is an open set $U$ in $D$ if $f^{2}(M)$ such that for every $f \in U$ and for any $\beta \in(0,1]$, the space of $\beta$-Hoelder cocycles of $f$ is $C^{0}$-stable, and the subspace of cocycles cohomologous to a constant is the common zero set of the periodic cycles functionals, i.e., $\phi \in L_{\beta}$ is cohomologous to a constant, with $C^{0}$ transfer function, if and only if $F(C)(\phi)=0$ for all periodic cycles $C$.

Moreover, $U$ contains all time-one maps for contact Anosov flows on $M$.

Actually, it follows easily from the arguments above that: 
Proposition 5.3. Proposition 5.2 and Theorem 5.2 are true in a slightly more general form. Namely, they hold for small enough perturbations of any partially hyperbolic diffeomorphism $g$ of a compact manifold such that

(1) the neutral foliation of $g$ is smooth and one-dimensional;

(2) the pair $\left(W^{s}, W^{u}\right)$ is locally transitive.

Also, we would like to mention that the transitivity of stable and unstable foliations is a key property in the study of stable ergodicity of partially hyperbolic diffeomorphisms. The stable ergodicity was established for the time-one map of the geodesic flow on a surface of constant (Grayson, Pugh, Shub in [6]) and variable (Wilkinson in [19]) negative curvature. Pugh and Shub ([18]) proved the stable ergodicity for a class of algebraic diffeomorphisms of homogeneous spaces. Brezin and Shub ([1]) proved the stable ergodicity of time one map of the geodesic flow on a compact manifold of constant negative curvature.

Our results, together with the results of Pugh and Shub ([18]), imply the stable ergodicity of the time-one maps of the geodesic flows on the compact manifolds of variable negative curvature (with suitable restrictions on the pinching of the curvature).

\section{Stability of $C^{\infty}$-cocycles}

As we have pointed out if the cocycle is $C^{\infty}$ and the periodic cycles functionals vanish then the transfer function constructed in Theorem 3.1 has continuous derivatives of all orders along the stable and unstable foliations.

Therefore, to prove the regularity of the transfer function we need some result which would guarantee the smoothness of functions which are smooth along "sufficiently many directions." We use the following powerful Hermander-type theorem (see [11], Theorem 2.1, where it is deduced from a number of results published elsewhere).

Theorem B 6.0.1. Let $\mathcal{D}_{1}, \ldots, \mathcal{D}_{k}$ be $C^{\infty}$ distributions on a manifold $M$ such that their sum is totally non-integrable. Also, assume that for each $j$, the dimension of the space spanned by the commutators of length at most $j$ at each point is constant in a neighborhood.

Let $P$ be a function, or even a generalized function or distribution, on $M$. Suppose that for any positive integer $p$ and $C^{\infty}$ vectorfield $X$ tangent to any $\mathcal{D}_{j}$, the $p$-th partial derivative $X^{p}(P)$ exists as a continuous or a local $L^{2}$ function, then $P$ is $C^{\infty}$ on $M$.

Thus, we immediately have (from Theorems 3.1 and B) the following: 
Theorem 6.1. If $f$ is a partially hyperbolic diffeomorphism of a compact manifold such that the foliations $W^{s}$ and $W^{u}$ are $C^{\infty}$ and the pair $\left(W^{s}, W^{u}\right)$ is totally non-integrable, then the space of $C^{\infty}$-cocycles of $f$ is $C^{\infty}$-stable, and the subspace of cocycles cohomologous to a constant is the common zero set of the periodic cycles functionals.

In particular we have the following

Corollary 6.1. For all transformations described in Corollary 4.1 the spaces of $C^{\infty}$-cocycles are $C^{\infty}$-stable, and the subspaces of cocycles cohomologous to a constant are the common zero sets of the periodic cycles functionals.

Recently R.de le Llave (private communication) was able to extend Theorem B to certain cases when the distributions themselves are not necessarily smooth, but their sum is still smooth. In particular, his results apply in the setting of Section 5, so one has the following

Corollary 6.2. For the time-one map of a contact Anosov flow (in particular, for a geodesic shift $g^{1}$ on a compact manifold of negative curvature) the space of $C^{\infty}$-cocycles is $C^{\infty}$-stable, and the subspace of cocycles cohomologous to a constant is the common zero set of the periodic cycles functionals.

\section{Concluding remarks}

Theorems 3.1, 3.2 and 6.1 can easily be reformulated and reproved for partially hyperbolic flows. Corollaries $3.1,4.1,4.2$, and 6.1 remain true for flows. (Of course, in the statements of Corollaries 4.1 and 6.1 we need to replace the single transformation generated by $a \in A$ by the flow generated by the one parameter subgroup of $A$ that contains $a$.)

An even more interesting generalization is to twisted cocycles. Namely, we can prove the analogs of Theorems 3.1 and 3.2 for twisted cocycles with coefficients in $\mathbb{R}^{k}$, twisted by "slow" cocycles with coefficients in $G L(k, \mathbb{R})$. (A cocycle $\alpha$ is called slow if for all $x$ and $n,\left\|\alpha\left(f^{n}(x)\right)\right\| \leq C e^{|n| \lambda}$, where $C$ is a constant and $\lambda<\min \left\{\lambda_{1}, \lambda_{2}\right\}$. Here $\lambda_{1}$ and $\lambda_{2}$ are as in Definition 3.1.) Such twisted cocycles arise in connection with the infinitesimal conjugacy problems.

In all likelihood, Theorems 3.1 and 3.2 can be extended to cocycles with much more general coefficients. Namely, to Lie groups with the two-sided metrics (like in [13]) and other finite and even infinite dimensional Lie groups (like in [17]).

Also, notice that our results for Weyl chamber flows are complementary to the results of Katok and Spatzier in [11]. To be more precise, the 
results from [11] describe the cocycles for the actions of subgroups of $A$, of dimension bigger than one, on $K \backslash G / \Gamma$, while Corollaries 4.1 and 6.1 give us a description of the cocycle spaces for the actions of one-parameter subgroups and individual elements $a \in A$.

Let us point out the importance of the transitivity conditions in Theorems 3.1, 3.2 and 6.1. Veech ([20]) shows that for partially hyperbolic automorphisms of a torus the periodic conditions form a generating set of cohomological obstructions. In particular, it implies that if the periodic conditions vanish then our periodic cycles functionals vanish as well. Veech has shown ([20], Proposition 1.5) that for partially hyperbolic, but not hyperbolic, automorphisms of a torus the spaces of $C^{1}$-cocycles are not $C^{1}$-stable . Most likely one can construct examples of partially hyperbolic automorphisms of a torus with $C^{0}$-non-stable spaces of Hoelder cocycles.

And finally we would like to formulate two open problems:

1. Will Theorems 3.1 and/or 3.2 be true if we replace in their assumptions the periodic cycles functionals by $f$-invariant measures?

2. Will Corollaries 4.1 and 5.1 be true if we replace in their assumptions the periodic cycles functionals by, respectively, the $g^{t}$-invariant and $A$ invariant measures?

\section{Acknowledgments}

We would like to express out sincere gratitude to M.Brin and Ya.Pesin for helpful discussions of the transitivity property for various partially hyperbolic systems. We also would like to thank A.Wilkinson who read a preliminary version of the paper and made several useful comments.

\section{References}

1. J. Brezin and M. Shub, Stable Ergodicity in Homogeneous Spaces, preprint.

2. M. I. Brin and Ya. B. Pesin, Partially hyperbolic dynamical systems, Math. of USSR., Izvestia 8, no.1, (1974), 177-218.

3. M. I. Brin, Topological transitivity of one class of dynamical systems and frame flows on manifolds of negative curvature, Funct. Anal. and its Applic. 9, (1975), 8-16.

4. P. Collet, H. Epstein, and G. Gallavoti, Perturbations of geodesic flow on surfaces of constant negative curvature and their mixing properties, Comm. Math. Phys. 95, (1984), 61-112.

5. G. Forni, Solutions of the cohomology equation for area-preserving flows on compact Riemann surfaces, ERA Amer. Math. Soc. 1 (1995), 114-123.

6. M. Grayson, C. Pugh, and M. Shub, Stably ergodic diffeomorphisms, Ann. of Math. 140 (1994), 295-329.

7. V. Guillemin and D. Kazdan, On the cohomology of certain dynamical systems, Topology 19 (1980), 291-299.

8. ___ Some inverse spectral results for negatively curved 2-manifolds, Topology 19 (1980), 301-313. 
9. M. Hirsh, C. Pugh, and M. Shub, Invariant manifolds, Lec. Notes Math. 583, Springer-Verlag, Berlin, (1985).

10. A. Katok and B. Hasselblatt, Introduction to the modern theory of dynamical systems, Cambridge Univ. Press, (1995).

11. A. Katok and R. J. Spatzier, Subelliptic estimates of polynomial differential operators and applications to rigidity of abelian actions, Math. Res. Let. 1 (1994), 193-202.

12. A. Katok in collaboration with E. A. Robinson, Constructions in ergodic theory, preprint.

13. A. Livsic, Cohomology of dynamical systems, Math. USSR., Izvestia 6 (1972), 12781301.

14. __ Homology properties of U-systems, Math. Notes 10 (1971), 758-763.

15. R. de la Llave, J. M. Marco, and R. Moryion, Canonical perturbation theory of Anosov systems and regularity results for Livsic cohomology equation, Ann. of Math. 123 (1986), 537-612.

16. R. de la Llave, Analytic regularity of solutions of Livsic's cohomology equation and some applications to analytic conjugacy of hyperbolic dynamical systems, preprint, (1989).

17. V. Nitica and A. Torok, Cohomology of dynamical systems and rigidity of partially hyperbolic actions of higher rank lattices, Duke J. of Math. 79 (1995), 751-810.

18. C. Pugh, M. Shub, Stably Ergodic Dynamical Systems and Partial Hyperbolicity, preprint.

19. A. Wilkinson, Thesis - University of California, Berkeley, 1995.

20. W. A. Veech, Periodic points and invariant pseudomeasures for toral endomorphisms, Ergodic Theory and Dynamical Systems 6 (1986), 449-473.

Department of Mathematics, The Pennsylvania State University, UniverSITY PARK, PA 16802

E-mail address: katok_a@math.psu.edu

Department of Mathematics, The Pennsylvania State University, UniverSITY PARK, PA 16802

E-mail address: avk@math.psu.edu 